\title{
TRACE DRIVEN REGISTRATION OF NEURON CONFOCAL MICROSCOPY STACKS
}

\author{
Luke Hogrebe ${ }^{1,2}$, Antonio R. C. Paiva ${ }^{1}$, Elizabeth Jurrus ${ }^{1,3}$, Cameron Christensen ${ }^{1}$ \\ Michael Bridge ${ }^{4}$, J.R. Korenberg ${ }^{4}$, Tolga Tasdizen ${ }^{1,2}$ \\ ${ }^{1}$ Scientific Computing and Imaging Institute, ${ }^{2}$ Dept. of Electrical and Computer Eng. \\ ${ }^{3}$ School of Computing, ${ }^{4}$ Brain Institute \\ University of Utah, Salt Lake City, UT, USA
}

\begin{abstract}
Active research in the area of 3-D neurite tracing has predominantly focused on single sections. Ultimately, however, neurobiologists desire to study the long range connectivity of the brain, which requires tracing axons across multiple serially-cut sections. Registration of axonal sections is challenging due to several factors, such as sparseness of the axons and complications of the sectioning process, including tissue deformation and loss. This paper investigates a method for registering sections using centerline traces which provide the locations of axons at section boundaries and the angles at which the axons approach the boundaries. This information is used to determine correspondences between two serial sections. Both global and local differences are accounted for using rigid and non-rigid transforms. Results show that utilizing information from traced axons allows axon continuity across sections to be restored.
\end{abstract}

Index Terms - Serial section registration, Axonal section registration, Non-rigid point registration.

\section{INTRODUCTION}

Tracing axonal projections across large distances (several cubic centimeters) is necessary for constructing a connectivity diagram of the brain. Senft [1] highlighted the importance of long axonal projections with examples in mice, noting that activity in one region of the brain can significantly influence behavior in another through only a sparse network of far traveling axons. Any attempts at generating digital reconstructions of these axonal networks require histological sectioning. The sectioning/preparation process introduces several deformations, however, including tissue shrinking and wrinkling as well as distortion induced by the microtome [2]. These deformations disrupt axon continuity across sections. This work investigates the problem of section-to-section registration with the goal of restoring proper axon continuity. Note that we refer to a z-stack as the optical slices captured by a single field of the microscope and to a set of mosaicked z-stacks as a section.

The images used in this work are produced by selectively staining axons exhibiting genes of interest (see Section 3.1). Consequently, our data is relatively sparse, and intensity based registration algorithms are not easily applicable. In this paper, we instead assume that traces of axons within sections are available to aid in section registration. A large body of research exists for tracing within a section and can be used for this purpose [3,4]. Under this assumption one piece of vital information available is the locations of axons at the

This work was supported under NIH grant 1RC1NS069152-01: A Computational Framework for Mapping Long Range Genetic Circuits. section boundaries. These provide the landmarks used for the registration. The landmark-based registration paradigm is commonly seen in remote sensing, fingerprint, and medical registration applications where the objective is to align a sensed image to a reference image given a set of landmarks [5]. The challenge arises due to the unknown correspondences between landmarks in the sensed (moving) and reference images. Another complication is the lack of oneto-one correspondences between the landmarks of the unregistered images due to inherent variability in landmark detection. Our landmarks are dependent on how well axons are traced. For this paper, we attempt to simplify the problem by obtaining axon annotations manually, but even then one-to-one correspondences are not always observed because of human subjectivity.

Current literature specifically addressing section-to-section registration of axon segments per se is still in its early development. Oberlaender et al. [6] presented an entire framework for reconstruction of 3-D neuron morphology, but discussion on section splicing is contained to coarse alignment using automatically detected blood vessels. Our current datasets do not have a blood vessel channel, so these landmarks are unavailable. Transmission electron microscopy registration methods [7] are capable of utilizing intensities since the images contain dense information. Our datasets are predominantly dark background, which prevents the direct use of correlation based techniques. One application that has influenced this work is automated fingerprint identification systems due to the association of orientations with landmarks as well as similarities in the obstacles encountered, including lack of one-to-one landmark correspondences and non-linear image deformations $[8,9]$.

\section{METHODS}

We propose a method for finding axon correspondences across section boundaries using axon locations as well as the angles of incidence at the boundaries. The correspondences chosen dictate the stack transformations, so we aim to maximize the correspondence accuracy. A rigid transform accounts for global rotation and translation differences between two sections based on a rough initial assignment of axon correspondences. The correspondences are then updated, followed by a non-rigid transformation to restore axon continuity.

\subsection{Axon Trace Information}

While automated neurite tracing algorithms continue to undergo active study, in this paper we assume axons within the unregistered sections have been traced to constitute the landmark detection step for registration. For the results presented in Section 3 the tracing is 


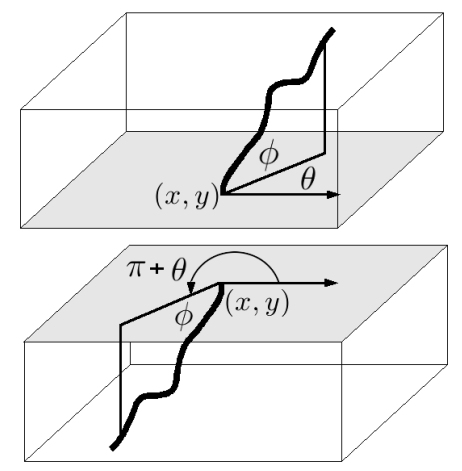

Fig. 1. Angle information for a traced axon across a section boundary.

performed using the semiautomated live-wire approach [10] conceptually similar to the scheme presented in [11].

In addition to the locations where an axon exits a section, $(x, y)$, the two angles obtained from the axon traces, $\phi$ and $\theta$, are depicted in Fig. 1. The z-axis angle is denoted by $\phi$ and represents how steep the axon approaches the top or bottom of the section. The vector used to determine $\phi$ is defined by an average of the coordinates of tracing data points nearest the stack boundary to account for axon tortuosity at the boundary. The angle $\theta$ describes the orientation of the axon in the $x-y$ plane and is obtained from the projection of same vector used to calculate $\phi$ onto the $x-y$ plane. While it is true that the aforementioned tissue distortions likely affect these angles, empirical evidence suggests that it is still valid to use them for registration.

\subsection{Coarse Alignment}

We first correct for any global rotation and translation of the moving dataset. Mathematically, for coarse alignment we are looking to find the rotation matrix and translation vector of the model [12],

$$
\mathbf{p}_{\text {reference }}^{(i)}=\mathbf{R} \mathbf{p}_{\text {moving }}^{(i)}+\mathbf{T}+\boldsymbol{\eta}^{(i)}
$$

where $i \in 1, \ldots, N$ points, $\mathbf{p}_{\text {reference }}^{(i)}$ is the $i^{\text {th }} 2 \times 1$ reference point vector, $\mathbf{R}$ is a $2 \times 2$ rotation matrix, $\mathbf{p}_{\text {moving }}^{(i)}$ is the $i^{\text {th }} 2 \times 1$ moving point vector, $\mathbf{T}$ is a $2 \times 1$ translation vector, and $\boldsymbol{\eta}^{(i)}$ is the $i^{\text {th }} 2 \times 1$ noise vector. The noise for our system is overwhelmingly due to local tissue deformations which are not captured by the rigid transformation.

\subsubsection{Correspondence Criterion}

Although the exact correspondences of the landmarks between datasets are unknown, we first make a rough guess based upon features of the local neighborhoods of the landmarks. Note that at this stage absolute spatial locations of landmarks can not be used due to the unknown global translation. Similarly, the absolute $\theta$ angles can not yet be used since we have not accounted for the potential rotation of the moving dataset. Therefore, we make use of relative location information in local neighborhoods. A local neighborhood with respect to a given reference landmark refers to its $k$-nearest neighbors. The features of each neighborhood include the Euclidean distance from each neighbor to the reference landmark and the relative angles between the neighbors (see Fig. 2). The neighbor

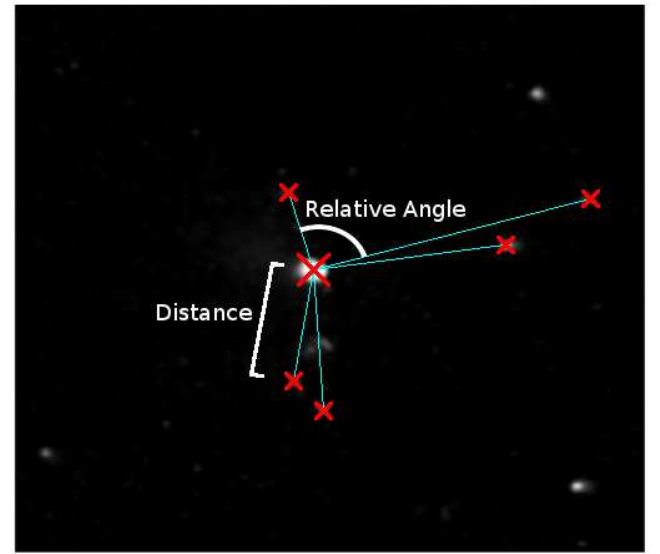

Fig. 2. Example local neighborhood consisting of five nearest neighbors for the large diameter axon in the center (reference landmark). As previously noted most of the intensity information for our data is dark background.

closest to the reference landmark is used as the starting point when ordering the distances and relative angles of the neighbors. The objective when selecting characteristics for local neighborhoods is to ensure invariance to rotation and translation [8]. The $\phi$ angles of the landmarks themselves are also included as a feature.

The features for a given landmark are compared against those of landmarks of the opposite set to determine landmark similarities. Each potential correspondence measure, $\gamma$, is obtained by weighting the absolute value of the sum of differences between each of the feature components and summing the result (2).

$$
\begin{aligned}
\gamma_{m, n}=\omega_{1}\left(\sum_{z=1}^{k_{s}}\left|D_{m, z}-D_{n, z}\right|\right) & +\omega_{2}\left(\sum_{z=1}^{k_{s}}\left|\angle_{m, z}-\angle_{n, z}\right|\right) \\
& +\omega_{3}\left(\left|\phi_{m}-\phi_{n}\right|\right)
\end{aligned}
$$

where $m$ and $n$ are the indices of landmarks of the two sets, $k_{s}$ is the number of nearest neighbors in the same set for a given landmark, $\omega_{1: 3}$ are weights, $D_{x, z}$ is the Euclidean distance to the $z^{t h}$ neighbor with respect to reference landmark $x, \angle_{x, z}$ is the angle between the $z^{t h}$ and counter clockwise $z^{t h}+1$ neighbors for landmark $x$, and $\phi_{x}$ is the angle of approach for landmark $x$.

A limitation of this criterion is that local neighborhoods are highly sensitive to points with no corresponding landmarks, meaning a stray landmark can cause the local neighborhood features to be drastically different for truly corresponding landmarks. Following the initial correspondence assignments, Section 2.2.3 addresses how incorrect correspondences are handled.

\subsubsection{Weighted Bipartite Matching}

After the feature vectors for each landmark are compared by (2), correspondences must be assigned. Weighted bipartite matching is applicable to the problem of landmark correspondence assignments because the matching produces one-to-one correspondences [8]. A bipartite graph, $G=\{U, V, E\}$, consists of two disjoint sets, $U$ and $V$, whose edges, $E$, only link points belonging to different sets. Our two sets consist of the landmarks of the sections to be registered. A weighted graph is used with the graph edge costs given by the 
correspondence criterion in (2). Therefore, landmarks with similar local neighborhoods have a low cost edge link. The Hungarian algorithm [13] is used to find the minimum cost across all of the potential assignments.

\subsubsection{RANSAC}

As expected, the potential correspondences returned from the weighted bipartite matching are predominantly incorrect since the correspondence criterion is based on a landmark's local neighborhood and many neighborhoods are contaminated by points with no corresponding landmark in the adjacent section. However, the few correctly associated landmarks can be used to find a suitable leastsquares solution to the rotation and translation parameters using the RANdom SAmple Consensus algorithm (RANSAC) [14].

The purpose of RANSAC is to fit a given type of transformation model to a set of data containing outliers such that the number of inliers is maximized under the model. We aim to find the rotation and translation parameters of the model in (1) based on $\mathbf{p}_{\text {reference }}$ and $\mathbf{p}_{\text {moving }}$ landmarks that truly correspond in the presence of many false correspondences. To determine the goodness of a fit the method by Arun et al. [12] is used. This approach decouples the rotation and translation to efficiently find the least-squares solution for $\mathbf{R}$ and $\mathbf{T}$.

\subsection{Correspondence Refinement and Non-Rigid Transforma- tion}

Once the sections are roughly aligned, the use of a non-rigid transform is necessary for maximal restoration of axon continuity. The correspondences determine the source and destination landmarks for the non-linear warping and must be updated prior to the transformation. Two significant features are incorporated into the correspondence criterion. Accounting for the global rotation establishes relevance for the absolute $\theta$ angle shown in Fig. 1 since the $x-y$ axes now share the same zero angle reference. This angle adds axon identifying information because axons should generally exit one section and enter the next roughly $180^{\circ}$ apart as observed in our data. The second new feature available is the spatial locations of landmarks since the sections are roughly aligned. The implication is that correspondence comparisons can now be made against a greatly reduced set of landmark neighbors of the opposite section. Each new potential correspondence measure, $\Gamma$, is calculated as

$$
\Gamma_{m, n}=\omega_{4} \xi_{m, n}+\omega_{5}\left(|| \theta_{m}-\theta_{n}|-\pi|\right)+\omega_{6}\left(\left|\phi_{m}-\phi_{n}\right|\right)
$$

if the $n^{t h}$ landmark is a $k_{o}-\mathrm{NN}$ of $m$, and infinity otherwise. Variables $m, n, \omega$, and $\phi_{x}$ remain defined as before. The $\xi_{m, n}$ parameter is the Euclidean distance from landmark $m$ to a $k_{o}$-NN landmark $n$, and $\theta_{x}$ is the $\mathrm{x}$-y plane boundary angle of approach for landmark $x$.

Weighted bipartite matching is again used to designate correspondences. A difference from its previous use is that for a given node in the bipartite graph, all but a landmark's nearest neighbor edge links are set to infinity, meaning certainty of no correspondence. This limits incorrect correspondences to be made regionally. A distance threshold is used to limit correspondences being made for outlier points, meaning if two points are deemed corresponding but are too far apart, their correspondence is revoked.

The non-rigid point transformation utilized is the thin-plate spline model. The common analogy equates the transform to bending regions of a thin metal plate as source landmarks move to target destinations. The updated correspondences define our source and

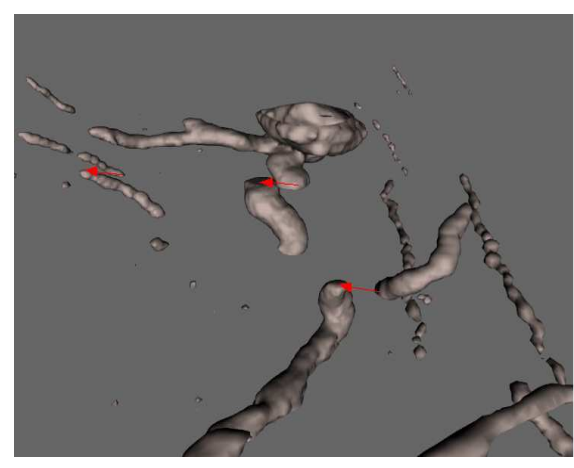

Fig. 3. Example region from Stacks 1 and 2 demonstrating why global corrections are not solely adequate for relinking separated axons. Red arrows indicate axon endpoints that should be connected.

destination landmarks, which we use to transform sections for final registration.

\section{RESULTS}

We traced axons in three serial sections, each consisting of four mosaicked $512 \times 512 \times 16 \mathrm{z}$-stacks using the live-wire based approach. These stacks will be referenced as Stacks 1 (top), 2 (center), and 3 (bottom). Acquisition details are provided in Section 3.1.

\subsection{Test Images}

Fluorescent microscopy z-stack mosaic acquisition was performed on 3 consecutive $\sim 22 \mu \mathrm{m}$ thick tissue sections over an area slightly anterior to the left periventricular nucleus region of the macaque brain. Sections underwent immunohistochemical staining for the neural fiber projection specific markers vasopressin, BEN (GTF2I), and oxytocin with Alexa 488, 568, and 647 labeled antibodies as fluorescent indicators, as well as DAPI counterstaining (cell nuclei). A Nikon A1R confocal microscope equipped for resonant mode imaging was used to capture at $40 X(0.62 \mu \mathrm{m} /$ pixel $)$ all 4 probe channels simultaneously. Acquisitions were performed over $11 \times 11 \mathrm{x}$-y fields with each field comprised of $\sim 25$ optical slices at $1.0 \mu \mathrm{m}$ intervals.

\subsection{Correspondence Accuracy}

For Stacks 1 and 2 the most inliers for any tested rigid model found by RANSAC which were correct one-to-one correspondences was 6 out of 35. For Stacks 2 and 3 RANSAC identified 6 out of 47 correct one-to-one correspondences. As previously noted, these numbers were expected to be low since the initial correspondence assignments were based on the landmarks' local features, which were susceptible to missing/extra landmarks. The significance is that RANSAC found good correspondences in the presence of mostly false assignments, a subset of which determined the rigid transformation parameters. Fig. 3 shows a region of roughly aligned Stacks 1 and 2. Local stretching distortion is visible here, demonstrating the need for a non-rigid transform.

Following global alignment we recomputed the correspondences incorporating the additional features discussed in Section 2.3. For Stacks 1 and 2 we were able to attain a correspondence accuracy of 33 out of 35 . Table 1 shows the correspondence correctness using nearest neighbor assignments for comparison. For Stacks 2 and 3 


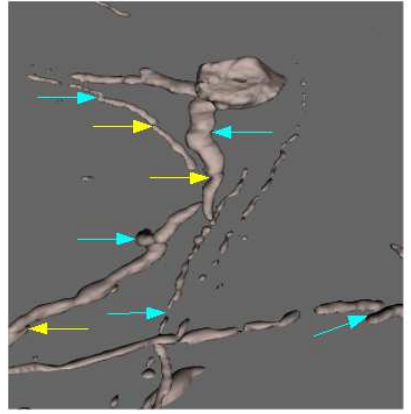

(a) Manual Alignment

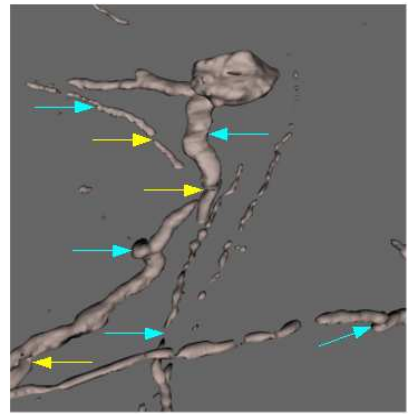

(b) Automated Alignment
Fig. 4. Example region showing the three sections aligned. (a) Alignment using a manual registration tool. (b) Alignment using the method presented following non-rigid transformations. Cyan arrows indicate transitions from Stack 1 to 2 . Yellow arrows indicate transitions from Stack 2 to 3 .

we reached 37 out of 47 correct correspondences. Using these correspondences, non-rigid transformations were computed to obtain the final registered sections. The incorrect correspondences introduced improper registration in small regions that required manual correction. Fig. 4 shows a region of the three stacks registered both manually and with the method investigated in this paper. Breaks in the renderings are the result of faint staining or black space above an axon before it was within a focal plane.

Table 1. Correspondence accuracies

\begin{tabular}{cccc}
\hline & $\begin{array}{c}\text { Before Rigid } \\
\text { Transform }\end{array}$ & \multicolumn{2}{c}{$\begin{array}{c}\text { Before Non-Rigid } \\
\text { Transform }\end{array}$} \\
\hline Stacks & & Nearest Neighbor & Our Method \\
\hline $1 \& 2$ & $6 / 35(17.1 \%)$ & $28 / 35(80.0 \%)$ & $33 / 35(94.3 \%)$ \\
$2 \& 3$ & $6 / 47(12.8 \%)$ & $31 / 47(66.0 \%)$ & $37 / 47(78.7 \%)$ \\
\hline
\end{tabular}

\section{CONCLUSIONS}

In this work we have investigated neural section registration by taking advantage of the explicit information available from axon trace data. We use locations and angles at which axons exit sections as features that describe potential landmark correspondences across different sections. Weighted bipartite matching assigns one-to-one correspondences. For coarse alignment RANSAC is used to find a global rotation/translation transformation. The thin-plate spline model is used as the non-rigid fine-tuning transformation needed to reestablish axon continuity.

In terms of future work, improving the axon-to-axon correspondence accuracy across sections is most notable. Incorporating an iterative refinement scheme for non-rigid transformations may be a plausible approach, where correspondences are recomputed at each iteration. A second extremely important goal is experimentation with larger mosaics and more sections, as is required to glean the desired information from tracking long range axon projections.

\section{REFERENCES}

[1] S. L. Senft, "Axonal navigation through voxel substrates: a strategy for reconstructing brain circuitry," in Computational Neuroanatomy: Principles and Methods, G. Ascoli, Ed., chapter 12. Humana Press, Totowa, New Jersey, 2002.

[2] J. Capowski, Computer Techniques In Neuroanatomy, Plenum Press, New York, 1989.

[3] A. Rodriguez, D. Ehlenberger, P. Hof, and S. Wearne, "Threedimensional neuron tracing by voxel scooping," J. Neuroscience Meth., vol. 184, pp. 169-175, 2009.

[4] K. Al-Kofahi, S. Lasek, D. Szarowski, C. Pace, G. Nagy, J. Turner, and B. Roysam, "Rapid automated threedimensional tracing of neurons from confocal image stacks," IEEE Trans. on Information Technology in Biomedicine, vol. 6, no. 2, pp. 171-187, 2002.

[5] A. Goshtasby, 2-D and 3-D Image Registration: For Medical, Remote Sensing, and Industrial Applications, John Wiley \& Sons, Inc., Hoboken, New Jersey, 2005.

[6] M. Oberlaender, R. Bruno, B. Sakmann, and P. Broser, "Transmitted light brightfield mosaic microscopy for threedimensional tracing of single neuron morphology," Journal of Biomedical Optics, vol. 12, no. 6, 2007.

[7] T. Tasdizen, P. Koshevoy, B. Grimm, J. Anderson, B. Jones, C. Watt, R. Whitaker, and R. Marc, "Automatic mosaicking and volume assembly for high-throughput serial-section transmission electron microscopy," J. Neuroscience Meth., vol. 193, pp. 132-144, 2010.

[8] C. Wen and T. Guo, "An efficient algorithm for fingerprint matching based on convex hulls," in Proc. Intl. Conf. on Computational Intelligence and Natural Computing (CINC), 2009, vol. 1, pp. 66-69.

[9] A. Bazen and S. Gerez, "Fingerprint matching by thin-plate spline modelling of elastic deformations," Pattern Recognition, vol. 36, pp. 1859-1867, 2003.

[10] E. Mortensen and W. Barrett, "Intelligent scissors for image composition," in Computer Graphics (SIGGRAPH '95), 1995, pp. 191-198.

[11] E. Meijering, M. Jacob, J.-C.F. Sarria, P. Steiner, H. Hirling, and M. Unser, "Design and validation of a tool for neurite tracing and amalysis in fluorescence microscopy images," $C y$ tometry Part A, vol. 58, pp. 167-176, 2004.

[12] K. Arun, T. Huang, and S. Blostein, "Least-squares fitting of two 3-d point sets," IEEE Trans. on Pattern Analysis and Machine Intelligence, vol. 9, no. 5, pp. 698-700, 1987.

[13] D. Jungnickel, Graphs, Networks, and Algorithms: Second Edition, Springer-Verlag Berlin Heidelberg, Germany, 2005.

[14] M. Fischler and Bolles, "Random sample consensus: A paradigm for model fitting with applications to image analysis and automated cartography," Commun. ACM, vol. 24, no. 6,1981 . 